\title{
Therapie von PatientInnen mit COVID-19
}

\author{
Handlungsempfehlung aus palliativmedizinischer Perspektive* \\ Management of Patients with COVID-19
}

Recommendations from a Palliative Care Perspective

\begin{abstract}
Autoren
Institute

1 Lungenklinik Heckeshorn, Klinik für Pneumologie, Helios Klinikum Emil von Behring, Berlin

2 LMU Klinikum, Klinik und Poliklinik für Palliativmedizin, München

3 Palliativmedizin und interdisziplinäre Onkologie, Med. Klinik III, St. Josefs-Hospital, Wiesbaden

4 Klinik für Pneumologie, Evangelische Lungenklinik, Berlin

5 Klinik für Palliativmedizin, Universitätsklinikum Bonn
\end{abstract}

W. Nehls ${ }^{1}$, S. Delis ${ }^{1}$, B. Haberland², B. O. Maier ${ }^{3}$, K. Sänger4, G. Tessmer ${ }^{4}$, L. Radbruch ${ }^{5}$, C. Bausewein ${ }^{2}$

Diese Handlungsempfehlung wurde erstellt durch die Deutsche Gesellschaft für Palliativmedizin (DGP) mit Unterstützung der Deutschen Gesellschaft für Pneumologie und Beatmungsmedizin (DGP e. V.).

online publiziert 21.4 .2020
Bibliografie

Pneumologie 2020; 74: 652-659

DOI 10.1055/a-1156-2759

ISSN 0934-8387

(c) 2020. Thieme. All rights reserved.

Georg Thieme Verlag KG, Rüdigerstraße 14,

70469 Stuttgart, Germany

Korrespondenzadresse

Prof. Dr. Claudia Bausewein PhD MSc

Direktorin der Klinik und Poliklinik für Palliativmedizin,

LMU Klinikum, Klinik und Poliklinik für Palliativmedizin,

Campus Großhadern, Marchioninistr. 15,

81377 München, Deutschland

claudia.bausewein@med.uni-muenchen.de

\section{Präambel}

Bei der COVID-19-Pandemie handelt es sich um eine sich sehr dynamisch entwickelnde Situation, die die palliativmedizinische Versorgung an verschiedenen Punkten berührt.

- Es gilt, auf eine Häufung von Sterbefällen mit den Leitsymptomen Atemnot und Angst im Bereich der Akutmedizin vorbereitet zu sein und die akutmedizinischen Strukturen in ihrer Kompetenz mittels klarer Handlungsempfehlungen und Beratung durch palliativmedizinische Spezialistlnnen entsprechend zu stärken (Handlungsempfehlung 1).

- Zudem muss die palliativmedizinische Versorgung im ambulanten und stationären Sektor trotz erschwerter Rahmenbedingungen aufrechterhalten werden (Handlungsempfehlung 2).

In der aktuellen Situation der COVID-19-Pandemie müssen viele Fragen der Therapiezielfindung, Indikationsstellung und Eruierung des Patientenwillens bei PatientInnen mit COVID-19-Erkrankung beantwortet werden.
Empfehlungen zu „Entscheidungen über die Zuteilung von Ressourcen in der Notfall- und der Intensivmedizin im Kontext der COVID-19-Pandemie“ wurden von der Arbeitsgemeinschaft Ethik in der Medizin in Zusammenarbeit mit medizinischen Fachgesellschaften (darunter der DGP) formuliert [1].

Die Herausforderung von Triage-Konzepten vor dem Hintergrund eingeschränkter intensivmedizinischer Behandlungskapazitäten auch mit der möglichen Konsequenz der Beendigung einer bereits begonnenen Intensivbehandlung aufgrund fehlender Erfolgsaussichten wird ausführlich in der Ad-hocEmpfehlung „Solidarität und Verantwortung in der CoronaKrise“ des Deutschen Ethikrats diskutiert [2].

Angesichts der zu erwartenden komplexen medizinethischen Dilemmata ist die Einbindung der palliativmedizinischen Expertise zur Festlegung von realistischen patientenzentrierten Therapiezielen sowohl bei der Entwicklung von Konzepten als auch bei konkreten Entscheidungen im Einzelfall sinnvoll und notwendig.

\footnotetext{
* Stand 30.03.2020.
} 


\section{Stationäre Aufnahme}

Unabhängig von der aktuell besonderen Gesundheitslage sollte bei jeder stationären Aufnahme vor Einleitung einer Maßnahme das Ausmaß der Therapieintensivierung festgelegt werden. Der/die aufnehmende Arzt/Ärztin soll bei allen PatientInnen eine Festlegung zur Therapieeskalation vornehmen (s. Grundsätze zur Therapieeskalation und zur Therapielimitation). Konkret müssen folgende Fragestellungen durch ärztliche Anordnung immer bei Beginn der Behandlung gut sichtbar beantwortet werden:

\section{ÜBERSICHT}

Fragestellungen, die immer bei Beginn der Behandlung gut sichtbar beantwortet werden müssen

- Intubation: $\square$ ja/ $\square$ nein

- Intensivstation: $\square$ ja/ $\square$ nein

- NIV/High-Flow:

$\square$ ja/ $\square$ nein

$\square$ ja/ $\square$ nein

Bezugnehmend auf Handlungsempfehlungen anderer Fachgesellschaften sind PatientInnen bei akuter respiratorischer Insuffizienz ohne eine vorab festgelegte Therapielimitation durch eine COVID-19-Erkrankung bei Verschlechterung der klinischen Situation und zunehmender respiratorischer Insuffizienz zu intubieren [3]. Bei PatientInnen mit vorliegender Therapiebegrenzung bezüglich einer invasiven Beatmung sollten frühzeitig Wünsche zur möglichen nichtinvasiven Atemunterstützung abgesprochen werden.

Diese Festlegung kann PatientInnen vor Therapiemaßnahmen bewahren, die vor dem Hintergrund einer schweren Komorbidität/Grunderkrankung nicht zielführend sind; sie hilft erkrankten PalliativpatientInnen, in ihrem Betreuungskontext zu verbleiben, und unterstützt die sinnvolle Verteilung von medizinischen Ressourcen.

Entscheidungen für oder gegen eine medizinische Maßnahme müssen immer sorgfältig abgewogen werden und stellen für den verantwortlichen Arzt/die verantwortliche Ärztin eine große ethische Herausforderung dar. Wir empfehlen allen Einrichtungen, sehr kurzfristig zu überprüfen, welche Möglichkeiten standortbezogen bestehen, die ÄrztInnen hierbei zu unterstützen. MitarbeiterInnen, die hierbei zusätzlich an den Brennpunkten (Notaufnahme, Isolationsstationen, Intensivstationen etc.) zur Entscheidungsfindung in der Pandemie eingesetzt werden könnten, sind Mitglieder des lokalen Ethikkomitees, PalliativmedizinerInnen, EthikberaterInnen und PsychologInnen. Hilfreich ist es, wenn diese Angebote möglichst kurzfristig und umfangreich für die Handelnden zur Verfügung stehen.

\section{Grundsätze der Entscheidung zur Therapie- eskalation und zur Therapielimitation}

Im Rahmen der Behandlung einer akuten COVID-19-Infektion mit akuter respiratorischer Insuffizienz muss ein Bewusstsein dafür bestehen, dass diese akute Erkrankung das terminale Ereignis einer schweren Komorbidität darstellen kann. Der Palliativversorgung mit dem Ziel der optimalen Linderung von belastenden Symptomen kommt in diesen Situationen eine besondere Bedeutung zu.

Zur Entscheidungsfindung über eine eskalierende Therapiemaßnahme sind folgende Grundsätze zu beachten (s.a. - Abb. 1) [4]:

\section{Merke}

Voraussetzung für eine therapeutische Maßnahme ist die medizinische Indikation, die durch den behandelnden Arzt/ die behandelnde Ärztin vor dem Hintergrund der aktuellen Kenntnisse über Vorerkrankungen gestellt wird.

Kann durch eine therapeutische Maßnahme ein Therapieziel nicht erreicht werden, ist diese Maßnahme nicht sinnvoll und soll dem/der Patientln nicht angeboten werden. Zu Behandlungsbeginn bedeutet dies in der praktischen Umsetzung, dass Inhalte von Vorausverfügungen zu erfragen und zu berücksichtigen sind. Die Festlegung über die Therapielimitierung muss bereits zu Beginn erfolgen und sollte mit dem/der Patientln und den Angehörigen - soweit möglich - besprochen werden.

\section{Empfehlungen zur Symptomkontrolle von PatientInnen mit Covid-19-Atemwegs- erkrankung - Krankheitsbild und führende Beschwerden}

Die Infektion mit COVID-19 ist ein akutes Krankheitsbild mit dem klinischen Bild einer Pneumonie und begleitender respiratorischer Insuffizienz. Typische Symptome sind daher Dyspnoe, Husten, Schwäche und Fieber, Geschmack- und Geruchseinschränkungen, Diarrhoen sowie ein inhomogenes Bild an weiteren Symptomen. Aber auch akute Angst und Panik, Unruhe und Delir werden bei diesen PatientInnen beschrieben.

\footnotetext{
Merke

PatientInnen, die sich aufgrund der respiratorischen Insuffizienz verschlechtern und möglicherweise versterben, zeigen unter Umständen ein sich schnell entwickelndes ARDS mit akuter und schwerer Atemnot und sehr starker Angst und Panik. Sollte bei der/dem betroffenen Patientln keine Therapieeskalation mittels invasiver Beatmung vorgenommen werden, ist eine schnelle Symptomkontrolle unbedingt notwendig.
}

Die Prognose dieser PatientInnen, bei denen auf eine invasive Beatmung verzichtet wird, liegt eher bei Stunden bis wenigen Tagen. 


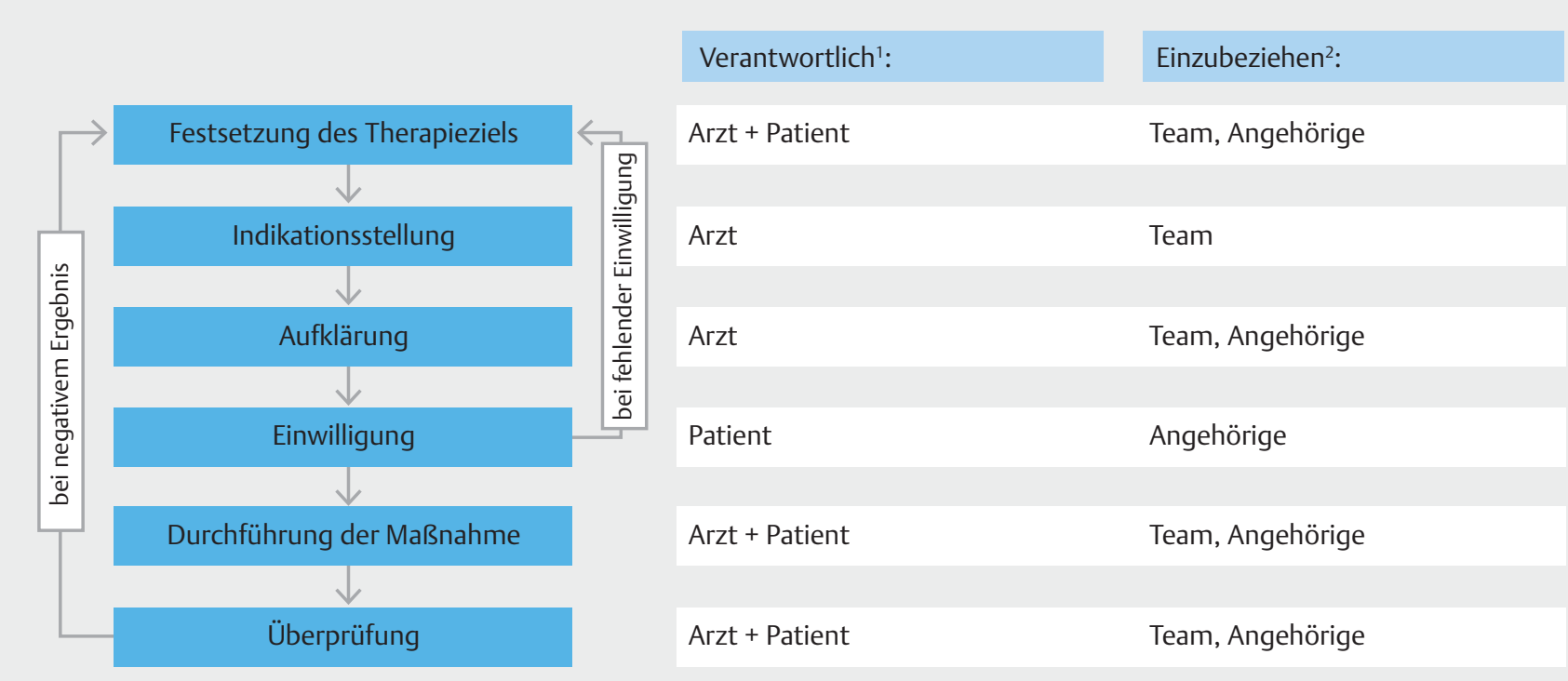

${ }^{1}$ Bei begründeten Zweifeln an der Einwilligungsfähigkeit des Patienten ist zusätzlich dessen Stellvertreter (Bevollmächtigter/ Betreuer) hinzuzuziehen. Dieser hat die Aufgabe, den Patienten im Prozess der Entscheidungsfindung zu unterstützen und bei Bedarf zu vertreten.

2 Sofern medizinisch sinnvoll bzw. vom Patienten gewünscht.

Abb. 1 Entscheidungsbaum zur Festlegung und Durchführung einer medizinischen Maßnahme (Quelle: S3-Leitlinie „Palliativmedizin für Patienten mit einer nicht heilbaren Krebserkrankung“) [rerif].

\section{Medikamentenapplikation}

\section{PRAXIS}

Vermeidung von Manipulationen im Nasen-RachenRaum

Aufgrund der hohen Konzentration von Viren im NasenRachen-Sekret sollten alle vermeidbaren Manipulationen im Nasen-Rachen-Raum bei COVID-19-PatientInnen oder PatientInnen mit Verdacht auf Corona-Infektion unterbleiben.

Das bedeutet auch, dass bei PatientInnen, die Unterstützung bei Medikamentengaben benötigen, unkontrolliert husten und Sekrete absondern, aus Gründen des Personal-/Selbstschutzes auf intranasale Applikationen von Medikamenten verzichtet werden sollte zugunsten einer primär oralen oder parenteralen Applikationsform.

\section{Symptomatische Therapie von therapierefraktärer Dyspnoe bei PatientInnen mit COVID-19}

Wenn Atemnot trotz optimaler Therapie der Akuterkrankung besteht, sollen nicht-medikamentöse und medikamentöse Maßnahmen zur Symptomkontrolle eingesetzt werden (s. S3-Leitlinie „Palliativmedizin“ [4]).

Bei milderen Verläufen mit mäßiger Atemnot können nichtmedikamentöse Maßnahmen wie Körperpositionen (Kutschersitz, Kissen unter die Arme etc.), Entspannung oder Küh- lung des Gesichts durch kühles Tuch (kein Handventilator wegen Aerosolverteilung!) zur Linderung der Atemnot beitragen.

Wenn eine Intensivtherapie und invasive Beatmung nicht indiziert sind, können Sauerstoffgabe und nasale High-FlowTherapie zu einer Symptomlinderung beitragen.

Wenn Atemnot trotz optimaler Therapie der Akuterkrankung besteht, sollen medikamentöse Maßnahmen zur Symptomkontrolle eingesetzt werden wie z. B. orale oder parenterale Opioide (s. S3-Leitlinie „Palliativmedizin“ [4]). Retardierte Opioide mit einem kontinuierlichen Wirkspiegel zeigen eine bessere Linderung der Atemnot als nicht-retardierte Formen. Allerdings ist eine rasche Dosistitration mit regelmäßigen kurzwirksamen Applikationen bei akuter Atemnot und sich rasch verschlechternden PatientInnen vorzuziehen.

Die kontinuierliche parenterale Gabe von Opioiden und Midazolam erfolgt i.d.R. s.c. oder i.v. über einen Perfusor. Wenn kein Perfusor verfügbar sein sollte, können die Medikamente auch alle $4 \mathrm{~h}$ i.v. als Kurzinfusion (KI) gegeben oder alle 4 h s.c. verabreicht werden (Dosierungen s. - Abb. 2; Cave: Bei einem Opioidwechsel [Rotation] immer Reduktion der dort angegebenen Dosierungen um 30 - 50\%!). Im ambulanten Setting können eine subkutane Dauernadel oder alternativ ButterflyNadel s.c. gelegt und die Angehörigen angeleitet werden, über die Nadel wiederholte Injektionen zu verabreichen. Die Perfusorgeschwindigkeiten zeigt $>$ Tab. 1 . 
Äquivalenzdosierungen zu Morphin

\begin{tabular}{|c|c|c|c|c|c|c|c|c|c|c|c|c|}
\hline \multicolumn{13}{|c|}{ Umrechnungstabelle Opioide } \\
\hline \multirow[b]{2}{*}{ Tilidin/Naloxon p.o. } & \multirow{2}{*}{\begin{tabular}{|l} 
Äquivalenzfaktor zu \\
Morphin i.v. \\
0,03
\end{tabular}} & \multirow[b]{2}{*}{100} & \multirow[b]{2}{*}{300} & \multirow[b]{2}{*}{600} & \multirow[b]{3}{*}{45} & \multirow[b]{3}{*}{60} & \multirow[b]{3}{*}{75} & \multirow[b]{3}{*}{90} & \multirow[b]{3}{*}{150} & \multirow[b]{3}{*}{225} & \multirow[b]{3}{*}{300} & \multirow[b]{3}{*}{450} \\
\hline & & & & & & & & & & & & \\
\hline Piritramid i.v. & 0,7 & 5 & 15 & 30 & & & & & & & & \\
\hline Morphin p.o./rektal & 0,3 & 10 & 30 & 60 & 90 & 120 & 150 & 180 & 300 & 450 & 600 & 900 \\
\hline Morphin s.c./i.v. & 1 & 3,3 & 10 & 20 & 30 & 40 & 50 & 60 & 100 & 150 & 200 & 300 \\
\hline Hydromorphon p.o. & 1,7 & 2 & 6 & 12 & 18 & 24 & 30 & 36 & 60 & 90 & 120 & 180 \\
\hline Hydromorphon s.c./i.v. & 5 & 0,7 & 2 & 4 & 6 & 8 & 10 & 12 & 20 & 30 & 40 & 60 \\
\hline
\end{tabular}

Bei einem Opioidwechsel (Rotation) immer Reduktion der hier angegebenen Dosierungen um 30-50\%!

Abb. 2 Äquivalenzdosierungen nach [5]. Cave: Bei einem Opioidwechsel (Rotation) immer Reduktion der hier angegebenen Dosierungen um $30-50 \%$ !

- Tab. 1 Perfusordosierungen in Abhängigkeit der vorbestehenden Morphin-Tagesdosis.

\begin{tabular}{|c|l|l|l|l|}
\hline Orale Morphin-Tagesdosis & Perfusordosierung & Konzentration & Laufgeschwindigkeit i.v. & $\begin{array}{c}\text { Entspricht ca. Tagesdosis } \\
\text { Morphin i.v./s.c. }\end{array}$ \\
\hline $50 \mathrm{mg}$ & $50 \mathrm{mg} / 50 \mathrm{ml} \mathrm{NaCl} 0,9 \%$ & $1 \mathrm{mg} / \mathrm{ml}$ & $0,6 \mathrm{ml} / \mathrm{h}$ & $14,4 \mathrm{mg} / \mathrm{d}$ \\
\hline $100 \mathrm{mg}$ & $100 \mathrm{mg} / 50 \mathrm{ml} \mathrm{NaCl} 0,9 \%$ & $2 \mathrm{mg} / \mathrm{ml}$ & $0,6 \mathrm{ml} / \mathrm{h}$ & $28,8 \mathrm{mg} / \mathrm{d}$ \\
\hline $150 \mathrm{mg}$ & $100 \mathrm{mg} / 50 \mathrm{ml} \mathrm{NaCl} 0,9 \%$ & $2 \mathrm{mg} / \mathrm{ml}$ & $1,0 \mathrm{ml} / \mathrm{h}$ & $48 \mathrm{mg} / \mathrm{d}$ \\
\hline $200 \mathrm{mg}$ & $100 \mathrm{mg} / 50 \mathrm{ml} \mathrm{NaCl} \mathrm{0,9 \%}$ & $2 \mathrm{mg} / \mathrm{ml}$ & $1,4 \mathrm{ml} / \mathrm{h}$ & $67,2 \mathrm{mg} / \mathrm{d}$ \\
\hline $250 \mathrm{mg}$ & $100 \mathrm{mg} / 50 \mathrm{ml} \mathrm{NaCl} \mathrm{0,9 \%}$ & $2 \mathrm{mg} / \mathrm{ml}$ & $1,8 \mathrm{ml} / \mathrm{h}$ & $86,4 \mathrm{mg} / \mathrm{d}$ \\
\hline $300 \mathrm{mg}$ & $200 \mathrm{mg} / 50 \mathrm{ml} \mathrm{NaCl} \mathrm{0,9 \%}$ & $4 \mathrm{mg} / \mathrm{ml}$ & $1,0 \mathrm{ml} / \mathrm{h}$ & $96 \mathrm{mg} / \mathrm{d}$ \\
\hline $400 \mathrm{mg}$ & $200 \mathrm{mg} / 50 \mathrm{ml} \mathrm{NaCl} \mathrm{0,9 \%}$ & $4 \mathrm{mg} / \mathrm{ml}$ & $1,4 \mathrm{ml} / \mathrm{h}$ & $134,4 \mathrm{mg} / \mathrm{d}$ \\
\hline
\end{tabular}

\section{Dosierungsempfehlungen}

Die Empfehlungen für Opioid-naive PatientInnen, bei denen eine Medikation p. o. möglich ist, fasst $>$ Tab. 2 zusammen; die entsprechenden Angaben für Opiod-vorbehandelte PatientInnen sind $\triangleright$ Tab. 3 zu entnehmen. Ist eine p. o.-Medikation nicht mehr möglich, sollten die in $>$ Tab. 4 dargestellten Behandlungsempfehlungen Anwendung finden.

\section{Symptomatische Therapie von Husten}

PatientInnen mit COVID-19-Atemwegserkrankung können sowohl durch trockenen als auch - bedingt durch bakterielle Superinfektion - produktiven Husten belastet sein.

Zu den allgemeinen Maßnahmen gehören ausreichende Luftfeuchtigkeit im Raum, orale Flüssigkeitsaufnahme, saure Bonbons lutschen, aufgerichteter Oberkörper beim Schlafen und pflanzliche Mittel (z. B. Thymian-Hustensaft $5-10 \mathrm{ml} / 8 \mathrm{~h}$ ).

Die medikamentösen Maßnahmen fasst $>$ Tab. 5 zusammen.
- Tab.2 Empfehlungen für Opioid-naive PatientInnen - Medikation p.o. möglich.

\begin{tabular}{|c|c|}
\hline Substanz & Dosierung \\
\hline *Morphin retardiert & $\begin{array}{l}10-0-10 \mathrm{mg}^{* *} \\
\text { (8.00 Uhr-0-20.00 Uhr) }\end{array}$ \\
\hline Magrocol Btl. & $1-0-0$ \\
\hline $\begin{array}{l}\text { ggf. Antiemetikum ergänzen: } \\
\text { Haloperidol }\end{array}$ & $\begin{array}{l}0,5-1 \mathrm{mg} \text { zur Nacht und bis } \\
\text { zu 2-stündlich }\end{array}$ \\
\hline \multicolumn{2}{|c|}{$\begin{array}{l}\text { + Bedarfsgaben symptomorientiert ggf. stündlich } \\
\text { (unretardierte Opioide; } 1 / 6 \text { der Tagesdosis): }\end{array}$} \\
\hline Morphin-Tropfen & $\begin{array}{l}2,5-5 \mathrm{mg}^{* *}(=2-4 \mathrm{gtt} \\
\text { Morphinlösung } 2 \%)\end{array}$ \\
\hline ggf. Morphin i.v. als KI/s.c. & $1-3 m g^{* *}$ \\
\hline
\end{tabular}


- Tab.3 Empfehlungen für Opioid-vorbehandelte PatientInnen - Medikation p. o. möglich.

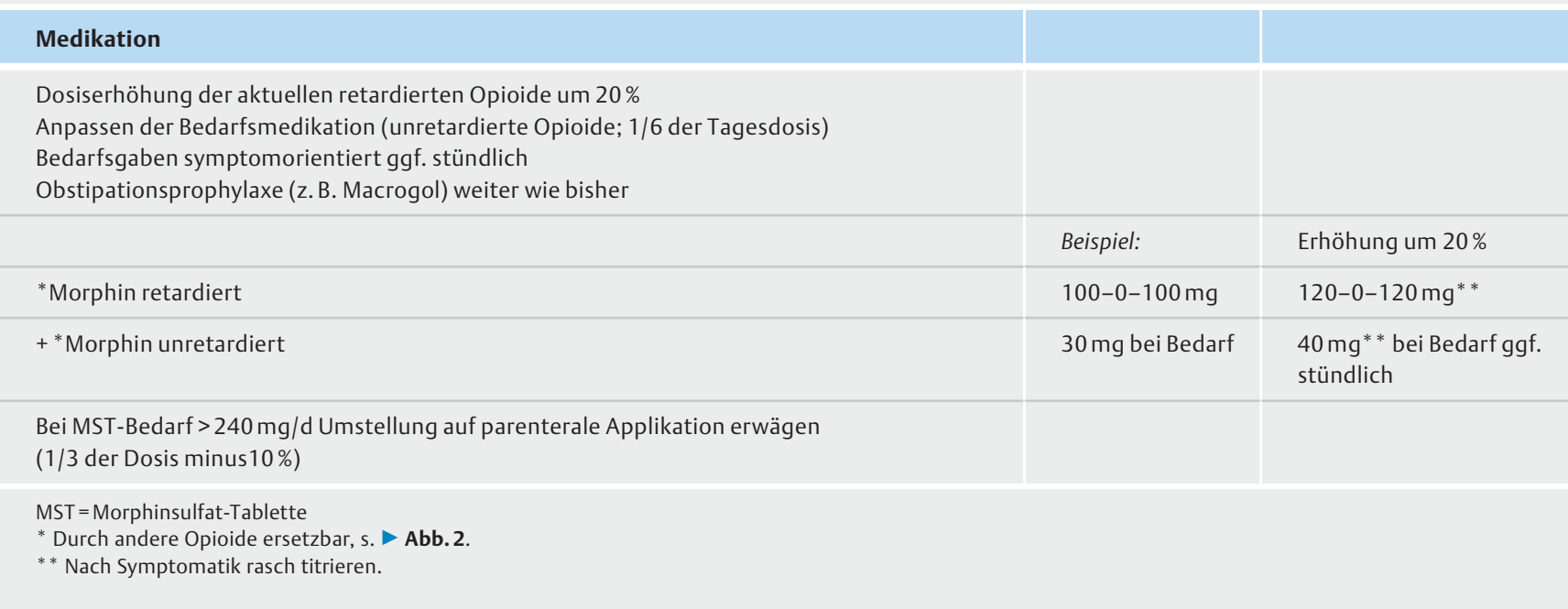

- Tab.4 Empfehlungen für COVID-19-PatientInnen - Medikation p. o. nicht mehr möglich.

\begin{tabular}{|c|c|c|}
\hline PatientInnen & Medikation & Beispiel \\
\hline \multicolumn{3}{|c|}{$\begin{array}{l}\text { Bei PatientInnen mit progredientem respiratorischem Versagen und Therapielimitation „keine Intubation/keine Reanimation“ ist es empfehlenswert, } \\
\text { parenterale Opioide zur Symptomkontrolle der therapierefraktären Dyspnoe frühzeitig anzusetzen. }\end{array}$} \\
\hline Opioid-naive PatientInnen & $\begin{array}{l}{ }^{* *} \text { Morphin } 5-10 \mathrm{mg} / 24 \mathrm{~h} \text { i.v./s.c. } \\
\text { z. B. } 50 \mathrm{mg}{ }^{*} \text { Morphin ad } 50 \mathrm{ml} \mathrm{NaCl} \mathrm{0,9 \% ,} \\
\text { Konzentration } 1 \mathrm{mg} / \mathrm{ml},{ }^{* *} \text { Start mit } 0,4 \mathrm{ml} / \mathrm{h}\end{array}$ & \multirow{2}{*}{$\begin{array}{l}\text { Beispiel: } \\
150-0-150 \mathrm{mg} \text { Morphin p. o. entspricht ca. } 100 \mathrm{mg} \\
\text { s. c. oder i. v./24 h } \\
200 \mathrm{mg}{ }^{*} \text { Morphin ad } 50 \mathrm{ml} \mathrm{NaCl} 0,9 \% \text {; Konzentration } \\
4 \mathrm{mg} / \mathrm{ml},{ }^{* *} \text { Start mit } 1 \mathrm{ml} / \mathrm{h}\end{array}$} \\
\hline Opioid-vorbehandelte PatientInnen & $\begin{array}{l}\text { Umstellung der bisherigen Opioide auf kon- } \\
\text { tinuierlich parenterale Gabe s.c. oder i.v. }\end{array}$ & \\
\hline
\end{tabular}

\section{Cave}

Bei produktivem Husten sollte insbesondere tagsüber möglichst von antitussiven Medikamenten Abstand genommen werden.

\section{Symptomatische Therapie von Rasselatmung}

In der Sterbephase tritt bei PatientInnen mit COVID-19-Atemwegserkrankung immer wieder Rasselatmung auf. Durch frühzeitige antisekretorische Therapie kann die Bildung von Sekretionen im Hypopharynx und der Trachea reduziert werden, bestehende Sekretionen werden dadurch aber nicht beeinflusst. Wiederholtes Absaugen führt genauso zur Verstärkung der Sekretionen wie parenterale Flüssigkeitszufuhr in der Sterbephase. Die symptomatische Therapie von Rasselatmung ist in - Tab. 6 dargestellt.

\section{Symptomatische Therapie von Unruhe/Angst}

Häufig ist Atemnot mit Unruhe, Angst- und Paniksymptomen verbunden. Bei PatientInnen mit respiratorischer Insuffizienz und Therapielimitation (keine Intubation/keine Reanimation) sollten Atemnot sowie Angstsymptomatik engmaschig erfasst und schnellstmöglich medikamentös behandelt werden.
- Tab. 5 Antitussive Therapie.

\begin{tabular}{|c|c|}
\hline Substanz & sierung \\
\hline Morphin & $\begin{array}{l}-5 \mathrm{mg} \text { p. o. } / 4 \mathrm{~h} \text { oder } 5-10 \mathrm{mg} / 24 \mathrm{~h} \text { kontinuierlich } \\
\text { c./i.v. }\end{array}$ \\
\hline Noscapin & $-50 \mathrm{mg}$ bis zu $3 \times$ tgl. \\
\hline \multicolumn{2}{|c|}{ Tab. 6 Symptomatische Therapie von Rasselatmung. } \\
\hline Substanz & Dosierung \\
\hline Butylscopolamin & $\begin{array}{l}\text { kontinuierlich s.c./i.v. } 40-80 \mathrm{mg} / 24 \mathrm{~h} \text { und } 20 \mathrm{mg} \\
\text { bei Bedarf symptomorientiert bis zu stündlich }\end{array}$ \\
\hline \multicolumn{2}{|l|}{ oder } \\
\hline Glycopyrronium & $\begin{array}{l}\text { kontinuierlich s. c./i.v. } 0,6-1,0 \mathrm{mg} / 24 \mathrm{~h} \text {, } \\
\text { bei Bedarf } 0,2 \mathrm{mg} \text { bis zu } 2 \text {-stündlich }\end{array}$ \\
\hline
\end{tabular}


Tab. 7 Symptomatische Therapie von Unruhe/Angst.

\begin{tabular}{|c|l|}
\hline Substanz & Dosierung \\
\hline Lorazepam & $\begin{array}{l}1 \mathrm{mg} \text { p. o./s. I. (ggf. mit } 2 \mathrm{ml} \text { Wasser auflösen) bei } \\
\text { Bedarf, symptomorientiert bis zu halbstündlich }\end{array}$ \\
\hline oder & \\
\hline Midazolam & $\begin{array}{l}2,5-5 \mathrm{mg} \text { i. v. als Kl/s.c. bei Bedarf, } \\
\text { symptomorientiert bis zu halbstündlich }\end{array}$ \\
\hline
\end{tabular}

- Tab. 8 Empfehlungen bei therapierefraktärer Angst und Unruhe.

\section{Maßnahmen}

- frühzeitige Umstellung auf parenterale Gabe i.v. (ggf. s.c.) kontinuierlich oder alle $4 \mathrm{~h}$

- Midazolam-Perfusor Anfangsdosis: Midazolam $10 \mathrm{mg} / 24 \mathrm{~h}$, (in Kombination mit nach Symptomatik titrieren, z. B. Morphin)

- $10 \mathrm{mg}$ Midazolam ad $50 \mathrm{ml}$ $0,9 \%$, Laufrate $2 \mathrm{ml} / \mathrm{h}$

- oder Midazolam 2,5-5 mg i.v. als $\mathrm{KI} / \mathrm{s}$. c. alle $4 \mathrm{~h}$
Empfehlungen zur medikamentösen Linderung von Angst und Unruhe, in Ergänzung zur Opioid-Therapie, sind in > Tab. 7 dargestellt. Empfehlungen bei therapierefraktärer Angst und Unruhe fasst $>$ Tab. 8 zusammen.

\section{Symptomatische Therapie von akuter Agitation und Delir}

Bedingt durch Infektion, Hypoxie, Isolationsmaßnahmen u.a. treten bei PatientInnen mit COVID-19-Infektionen häufig Agitation und Delir auf, die frühzeitig behandelt werden müssen. Mögliche weitere Ursachen für Agitation und Delir sollten abgeklärt und wenn möglich behandelt werden.

Zu den nicht-pharmakologischen Maßnahmen gehören neben Klärung und Behandlung möglicher Ursachen eine ausreichende Kommunikation und, wenn möglich, Ruhe (ruhiges und beleuchtetes Zimmer) sowie Orientierung für PatientInnen geben. Symptomatische medikamentöse Maßnahmen zeigt - Tab. 9.

- Tab. 9 Symptomatische Therapie von akuter Agitation und Delir.

\begin{tabular}{|c|c|c|}
\hline Substanz & \multicolumn{2}{|l|}{ Dosierung } \\
\hline \multicolumn{3}{|c|}{ Bei überwiegender motorischer Unruhe, z. B.: } \\
\hline Midazolam & \multicolumn{2}{|c|}{ 2,5-5 mg i.v. als KI/s. c. bei Bedarf, symptomorientiert bis zu halbstündlich } \\
\hline \multicolumn{3}{|l|}{ oder } \\
\hline Lorazepam & \multicolumn{2}{|c|}{ 0,5-1 mg s.l./p. o. bei Bedarf symptomorientiert bis zu halbstündlich } \\
\hline \multicolumn{3}{|l|}{ oder } \\
\hline Midazolam & \multicolumn{2}{|c|}{$\begin{array}{l}\text { kontinuierlich i. v. oder s. c. } 10 \mathrm{mg} / 24 \mathrm{~h} \\
\text { Beispiel: } 10 \mathrm{mg} \text { Midazolam ad } 50 \mathrm{ml} \mathrm{NaCl} 0,9 \% \text {, Laufrate } 2 \mathrm{ml} / \mathrm{h}\end{array}$} \\
\hline \multicolumn{3}{|c|}{ Bei Halluzinationen und Verwirrtheit: } \\
\hline Haloperidol & \multicolumn{2}{|c|}{1 - 2 mg s. c. bei Bedarf, symptomorientiert bis zu halbstündlich } \\
\hline \multicolumn{3}{|l|}{ oder } \\
\hline Haloperidol & \multicolumn{2}{|c|}{$\begin{array}{l}\text { s. c. kontinuierlich } 2-5 \mathrm{mg} / 24 \mathrm{~h} \\
\text { Beispiel: } 5 \mathrm{mg} \text { Haloperidol ad } 50 \mathrm{ml} \mathrm{NaCl} 0,9 \% \text {, Laufrate } 2 \mathrm{ml} / \mathrm{h}\end{array}$} \\
\hline \multicolumn{3}{|c|}{ - Tab. 10 Palliative Sedierung. } \\
\hline Medikament & Dosis & Kommentar \\
\hline Midazolam & $\begin{array}{l}\text { - Start z. B. mit } 10-20 \text { mg/24h s.c./i.v. } \\
\text { " ggf. initial Bedarfsgabe von } 1 \text { - } 5 \text { mg s. c./i. v. als KI } \\
\text { " Erhaltungsdosis i.d.R. } 20-60 \text { mg/24h s.c./i.v. }\end{array}$ & $\begin{array}{l}\text { ab } 60 \text { mg/24 h Ergänzung durch sedierendes Antipsychotikum, } \\
\text { z. B. Levomepromazin erwägen }\end{array}$ \\
\hline Levomepromazin & $\begin{array}{l}\text { - Start z. B. mit 12,5-25 mg/24h s.c./i.v. } \\
\text { " ggf. initial und während der Therapie Bedarfsgabe } \\
\text { von 5-25 mg, s. c./i.v. als KI, stdl. möglich } \\
\text { " Erhaltungsdosis i.d. R. 12,5-100 mg/24h } \\
\text { (Spannweite in der Literatur sehr groß) }\end{array}$ & $\begin{array}{l}\text { bei unzureichender Wirkung von Midazolam-Dosiserhöhungen } \\
\text { bzw. ab einer Midazolam-Dosis von ca. } 60 \mathrm{mg} / 24 \mathrm{~h}\end{array}$ \\
\hline
\end{tabular}




\section{Medikamentöse Symptomkontrolle bei COVID-19 und therapierefraktärer Dyspnoe}

Dauermedikation und Bedarfsmedikation nach Symptomatik rasch titrieren.

\begin{tabular}{|c|c|c|c|c|}
\hline \multirow[t]{2}{*}{ Symptom } & & \multicolumn{2}{|l|}{ Dauermedikation } & \multirow{2}{*}{$\begin{array}{l}\text { Bedarfsmedikation } \\
{ }^{* *} \text { symptomorientiert } \\
\text { ggf. halbstündlich }\end{array}$} \\
\hline & & $\begin{array}{l}\text { Retardpräparate p.o./oder } \\
\text { kontinuierlich als Perfusor } \\
\text { i.v./s.c. }\end{array}$ & $\begin{array}{l}\text { alle } 4 \text { Stunden p.o./i.v. als KI/s.c. } \\
\text { Alternative, wenn kein Perfusor } \\
\text { verfügbar }\end{array}$ & \\
\hline Atemnot & $\begin{array}{l}\text { Opioid-naiv } \\
\text { p.o. möglich }\end{array}$ & $\begin{array}{l}\text { *Morphin retardiert p.o. } \\
10-0-10 \mathrm{mg}\end{array}$ & $\begin{array}{l}\text { *Morphin Tropfen } 2-5 \mathrm{mg} \text { alle } 4 \mathrm{~h} \\
(=2-4 \mathrm{gtt} \text { Morphinlösung } 2 \%)\end{array}$ & *Morphin Tropfen 2-5 mg \\
\hline \multirow{2}{*}{$\begin{array}{l}\text { evtl. } \\
\text { Kombination } \\
\text { mit } \\
\text { Midazolam }\end{array}$} & $\begin{array}{l}\text { Opioid-naiv } \\
\text { i.v./s.c. Gabe } \\
\text { notwendig }\end{array}$ & $\begin{array}{l}\text { * Morphin } 5-10 \text { mg/24 h i.v./s.c. } \\
\text { Beispiel: } 50 \text { mg Morphin ad } \\
50 \text { ml NaCl 0,9\%, Konzentration } \\
\mathbf{1} \mathbf{~ m g / m l , ~}{ }^{*} \text { 'Start mit } \mathbf{0 , 4} \mathbf{~ m l} / \mathbf{h}\end{array}$ & $\begin{array}{l}\text { * Morphin } 1-3 \text { mg alle } 4 \text { h i.v. } \\
\text { als KI/s.c. }\end{array}$ & ${ }^{*}$ Morphin $1-3 \mathrm{mg}$ i.v. als KI/s.c. \\
\hline & $\begin{array}{l}\text { Opioid-vorbehandelt } \\
\text { i.v./s.c. Gabe notwendig } \\
\text { Beispiel: Morphin } \\
300 \mathrm{mg} / 24 \mathrm{~h} \text { p.o. }\end{array}$ & $\begin{array}{l}\text { Beispiel: } 200 \text { mg Morphin ad } \\
50 \mathrm{ml} \mathrm{NaCl} 0,9 \% \text {, Konzentration } \\
\mathbf{4} \mathbf{~ m g / m l , ~}{ }^{*} \text { Start mit } 1 \mathrm{ml} / \mathbf{h}\end{array}$ & $\begin{array}{l}\text { *Morphin } 15 \mathrm{mg} \text { alle } 4 \mathrm{~h} \text { i.v. als } \\
\text { KI/s.c. }\end{array}$ & $\begin{array}{l}\text { *Morphin } 10-15 \mathrm{mg} \text { i.v. } \\
\text { langsam über } 4 \mathrm{~h} \text { als KI/s.c. }\end{array}$ \\
\hline Husten & & *Morphin 5-10 mg/24 h s.c./i.v. & $\begin{array}{l}\text { *Morphin Tr. } 3-5 \text { mg p.o./4 h } \\
\text { Noscapin } 25-50 \text { mg bis zu } \\
3 \text { x tgl. }\end{array}$ & \\
\hline $\begin{array}{l}\text { Rassel- } \\
\text { atmung }\end{array}$ & & $\begin{array}{l}\text { Butylscopolamin s.c./i.v. } \\
40-80 \mathrm{mg} / 24 \mathrm{~h} \text { oder } \\
\text { Glycopyrronium s.c./i.v. } \\
0,6-1,0 \mathrm{mg} / 24 \mathrm{~h}\end{array}$ & & $\begin{array}{l}\text { Butylscopolamin } 20 \mathrm{mg} \\
\text { bis zu stündlich } \\
\text { Glycopyrronium } 0,2 \mathrm{mg} \\
\text { bis zu zweistündlich }\end{array}$ \\
\hline \multirow{2}{*}{$\begin{array}{l}\text { Unruhe/ } \\
\text { Angst } \\
\text { Kombination } \\
\text { mit Morphin }\end{array}$} & $\begin{array}{l}\text { orale Einnahme } \\
\text { möglich }\end{array}$ & $\begin{array}{l}\text { Lorazepam } 1 \text { mg s.l./p.o. (ggf. } \\
\text { in } 2 \text { ml Wasser auflösen) }\end{array}$ & $\begin{array}{l}\text { Lorazepam } 1 \text { mg s.I./p.o. } \\
\text { Midazolam 2,5-5 mg i.v. als } \\
\text { KI/s.c. }\end{array}$ & \multirow[t]{2}{*}{$\begin{array}{l}\text { Lorazepam } 1 \text { mg s.I./p.o. } \\
\text { Midazolam 2,5-5 mg i.v. als } \\
\text { KI/s.c. }\end{array}$} \\
\hline & $\begin{array}{l}\text { parenterale Gabe } \\
\text { notwendig }\end{array}$ & $\begin{array}{l}\text { Midazolam } 10 \text { mg/24 h i.v./s.c. } \\
\text { Beispiel: } 10 \text { mg Midazolam ad } \\
50 \mathrm{ml} \mathrm{NaCl} 0,9 \% \\
{ }^{* *} \text { Start mit } 2 \text { ml/h }\end{array}$ & $\begin{array}{l}\text { Midazolam 2,5-5 mg i.v. als } \\
\mathrm{KI} / \mathrm{s} . c \text {. alle } 4 \mathrm{~h}\end{array}$ & \\
\hline \multirow[t]{2}{*}{$\begin{array}{l}\text { Agitation \& } \\
\text { Delir }\end{array}$} & motorische Unruhe & $\begin{array}{l}\text { Midazolam } 10 \text { mg/24 h i.v./s.c. } \\
\text { Beispiel: } 10 \text { mg Midazolam ad } \\
50 \mathrm{ml} \mathrm{NaCl} \mathrm{0,9 \%} \\
{ }^{* *} \text { Start mit } 2 \mathrm{ml} / \mathrm{h}\end{array}$ & $\begin{array}{l}\text { Lorazepam } 1 \text { mg s.l./p.o. } \\
\text { Midazolam } 2,5-5 \text { mg i.v. als } \\
\text { KI/s.c. }\end{array}$ & $\begin{array}{l}\text { Lorazepam } 1 \text { mg s.I./p.o. } \\
\text { Midazolam 2,5-5 mg i.v. als } \\
\text { KI/s.c. }\end{array}$ \\
\hline & $\begin{array}{l}\text { Halluzinationen und } \\
\text { Verwirrtheit }\end{array}$ & $\begin{array}{l}\text { Haloperidol 2-5 mg/24 h s.c. } \\
\text { Beispiel: } 5 \text { mg Haloperidol ad } \\
50 \mathrm{ml} \mathrm{NaCl} \mathrm{0,9 \%} \\
{ }^{* *} \text { Start mit } 2 \text { ml/h }\end{array}$ & Haloperidol 1-2 mg s.c. alle $4 \mathrm{~h}$ & Haloperidol 1-2 mg s.c. \\
\hline
\end{tabular}

- Abb.3 Empfehlungen der Deutschen Gesellschaft für Palliativmedizin (DGP) zur medikamentösen Symptomkontrolle von PalliativpatientInnen mit COVID-19 und therapierefraktärer Dyspnoe.

*Morphin exemplarisch für andere Opioide, ggf. Umstellung nach entsprechenden Äquivalenzdosierungen, s. > Abb.2.

** Symptomorientierte Titration.

$\mathrm{KI}=$ Kurzinfusion

\section{Palliative Sedierung}

Bei ausgeprägter Atemnot mit Erstickungsängsten, Angst- und Unruhezuständen kann bei COVID-19-PatientInnen am Lebensende eine tiefe kontinuierliche Sedierung (palliative Sedierung) zur Symptomkontrolle und Ermöglichung eines friedlichen Sterbens notwendig sein. Dies ist insbesondere zu erwägen, wenn die o.g. Maßnahmen zur Linderung von Atemnot, Unruhe, Angst oder Delir nicht erfolgreich sind.

Die Durchführung einer palliativen Sedierung bei therapierefraktären Symptomen sollte gut dokumentiert sein: Nach Be- ginn der Sedierung sind Symptomlinderung, Sedierungstiefe mit Bewusstseinsniveau und Vigilanz regelmäßig zu evaluieren und zu dokumentieren.

Die entsprechenden medikamentösen Maßnahmen fasst - Tab. 10 zusammen.

Falls eine Sedierung mit den angegebenen Medikamenten nicht möglich ist, Kontakt zu spezialisierter Palliativmedizin (Palliativstation, Palliativdienst im Krankenhaus, SAPV) herstellen. 


\section{Zusammenfassung}

Die DGP-Empfehlungen zur medikamentösen Symptomkontrolle von PalliativpatientInnen mit COVID-19 und therapierefraktärer Dyspnoe zeigt $>$ Abb. 3 im Überblick.

Interessenkonflikt

Die Autorinnen/Autoren geben an, dass kein Interessenkonflikt besteht.

\section{Literatur}

[1] Deutsche Interdisziplinäre Vereinigung für Intensiv- und Notfallmedizin - DIVI. Entscheidungen über die Zuteilung von Ressourcen in der Notfall-und der Intensivmedizin im Kontext der COVID-19-Pan- demie. Im Internet (Stand: 11.04.2020): https://www.divi.de/empfehlungen/publikationen/covid-19/1540-covid-19-ethik-empfehlung-v2/file

[2] Deutscher Ethikrat. Solidarität und Verantwortung in der CoronaKrise. Ad-hoc-Empfehlung. Berlin: 2020. Im Internet (Stand: 11.04.2020): https://www.ethikrat.org/fileadmin/Publikationen/Adhoc-Empfehlungen/deutsch/ad-hoc-empfehlung-corona-krise.pdf

[3] Pfeifer M, Ewig S, Voshaar T et al. Positionspapier zur praktischen Umsetzung der apparativen Differenzialtherapie der akuten respiratorischen Insuffizienz bei COVID-19. 17. April 2020. (Stand: 19.04.2020): https://pneumologie.de/aktuelles-service/covid-19/ doi:10.1055/a-1157-9976

[4] Bausewein C, Voltz R, Radbruch L et al. Deutsche Krebsgesellschaft S3-Leitlinie Palliativmedizin für Patienten mit einer nicht heilbaren Krebserkrankung. AWMF-Registernummer: 128/001OL. Im Internet (Stand: 11.04.2020): https://www.dgpalliativmedizin.de/images/ stories/LL_Palliativmedizin_Langversion_1_1.pdf

[5] Bausewein C, Haberland B, Rémi C et al. Palliativmedizin pocketcard Set. Grünwald: Börm Bruckmeyer; 2016 